\title{
Propuesta de índices de gestión de servicios médico-quirúrgicos hospitalarios mediante técnicas estadísticas multivariantes
}

\author{
Hugo Salinas ${ }^{1,2}$, Alvaro Reyes ${ }^{3, a}$, Benjamín Carrasco ${ }^{4, b}$, \\ Patricio Veloz ${ }^{4, c}$, Marcia Erazo ${ }^{2, d}$, Sergio Carmona ${ }^{5}$, Luis \\ Martínez ${ }^{1}$.
}

\section{A proposal of new indices for hospital management}

Background: Diagnosis related groups (DRGs) are the most reliable patient classification system in hospital management. When this information is unavailable, other reliable classification system must be used. Aim: To obtain useful indices for hospital management, based on descriptive multivariate techniques. Material and Methods: Data on admissions to a University Hospital during 2003 were analyzed. Number of discharges, lethality rate, re-admission rate, number of outpatient consultations, length of hospital stay and surgical complexity index were analyzed, using information obtained by the Operations Management Department. The Principal Components Analysis (PCA) technique was applied and the R correlation matrix was used. Results: A total of 24,345 discharges were analyzed. The first two principal components were selected, accounting cumulatively for $76 \%$ of data variability ( $47 \%$ for the first and $29 \%$ for the second). Conclusions: The first component may be assimilated to a new index representing the difficulty of the attended cases, which we have termed Case Complexity. The second principal component would explain the number of attended persons, which we have termed Case Load. These two indices allow us to classify hospital services (Rev Méd Chil 2005; 133: 202-8).

(Key Words: Hospital administration; Management audit; Management, hospital financial)

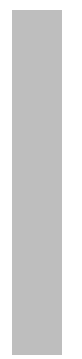

\footnotetext{
Recibido el 5 de agosto, 2004. Aceptado en versión corregida el 25 de noviembre, 2004.

${ }^{1}$ Departamento de Obstetricia y Ginecología, Hospital Clínico de la Universidad de Chile. ${ }^{2}$ Programa Doctorado en Salud Pública, Escuela de Salud Pública de la Universidad de Chile. ${ }^{3}$ Programa Magíster en Bioestadística, Escuela de Salud Pública de la Universidad de Chile. ${ }^{4}$ División de Operaciones, Hospital Clínico de la Universidad de Chile. ${ }^{5}$ Departamento de Obstetricia y Ginecología, Hospital Clínico San Borja Arriarán.

ainesiólogo

bIngeniero Civil Industrial

CIngeniero Civil Matemático

dEnfermera
}

Correspondencia a: Dr. Hugo Salinas P. Santos Dumont

999, Santiago, Chile. Fono Fax: (2) 7375325.

E-mail: hsalinas@ns.hospital.uchile.cl 
$\mathrm{T}$ oda la actividad que se realiza en el interior de los hospitales se encuentra condicionada por las características de los enfermos atendidos: edad, diagnóstico y tratamiento, entre otras. Medir dicha actividad ha sido una de las tareas más complejas para los gestores de salud, epidemiólogos clínicos y estadísticos, de tal forma que a lo largo de los años, han sido muchos los indicadores creados para dicho fin.

En la actualidad la actividad de un hospital se mide con indicadores clásicos, como número de egresos, días camas ocupadas, promedio de días de estada, tasa de letalidad por servicios, tasa de reingresos, etc. y se unen a estos indicadores otros que tienen en cuenta los casos atendidos, como son los derivados de los sistemas de clasificación de pacientes (SCP), siendo los grupos de diagnósticos relacionados (GDR), los que otorgan en la actualidad mayor confiabilidad ${ }^{1,2}$.

Los GDR, conforman un sistema de clasificación de pacientes, cuyo objetivo es obtener información respecto a episodios de enfermedad, recursos utilizados y manejo clínico realizado a dichos pacientes ${ }^{3}$. Los GDR han servido para definir indicadores que permitan comparar el funcionamiento global de los servicios médicos o médico-quirúrgicos de un hospital, siendo estos indicadores, el promedio de días de estada ajustados por funcionamiento, el promedio de días de estada ajustados por casos, el índice case mix y el índice funcional.

Para medir la actividad global de los servicios médicos o médico-quirúrgicos de un hospital, se deben analizar los indicadores mencionados para cada uno de los servicios, pero en muchos casos no es posible, por no contar con GDR en los establecimientos.

La información aportada por indicadores clásicos y los derivados de sistemas de clasificación de pacientes, permiten una comparación univariante clásica, donde en cada servicio médico o médicoquirúrgico, su indicador es una variable de comparación.

Esta forma de actuar no ofrece una visión multivariada de la actividad por servicios médicos o médico-quirúrgicos, no da un modelo de relación funcional entre los distintos indicadores e imposibilita obtener una clasificación de los servi- cios médicos y médico-quirúrgicos basada en sus actividades y en sus resultados.

El objetivo de este trabajo es generar nuevos índices de gestión de servicios médicos y médicoquirúrgicos hospitalarios, acordes a la realidad nacional y obtenidos dichos indicadores, generar índices mediante técnicas multivariantes, específicamente mediante el análisis de componentes principales (ACP). Dichos índices serán combinaciones lineales de los anteriormente descritos y permitirán establecer una clasificación de los servicios médicos y médico-quirúrgicos hospitalarios, que pueda explicar un modelo subyacente en el conjunto de la información que aportan los indicadores habituales.

\section{MATERIAL Y MÉTOdos}

Se estudiaron, durante el primer trimestre de 2004, los 24.345 egresos del Hospital Clínico de la Universidad de Chile ${ }^{4}$. Se desagregó la información de los servicios médicos y médico-quirúrgicos, desechando en esta etapa los primeros, por no contar con la posibilidad de obtener indicadores objetivos de complejidad más allá de las tasas de letalidad y reingresos.

Entre los servicios médico-quirúrgicos, se desecharon neonatología y máxilo facial, por no contar con actividad quirúrgica comparable y el Servicio de Emergencia, por ser un servicio de hospitalización transitoria, lo cual no permite obtener información de indicadores relevantes para el presente estudio. Para los 9 servicios médicos quirúrgicos restantes (cardiovascular, cirugía, neurocirugía, oftalmología, otorrinolaringología, traumatología, urología, ginecología y obstetricia), se midieron las siguientes variables:

1. Número total de egresos;

2. Tasa de letalidad, entendida como tasa de pacientes fallecidos por 1.000 egresos del servicio correspondiente, en el período de estudio;

3. Número de reingresos, definido como todo aquel ingreso, por el mismo diagnóstico, en los 10 días posteriores al alta; por 1.000 egresos; 
4. Número de consultas externas, definido como la frecuencia absoluta de consultas ambulatorias por cada servicio;

5. Número de días camas ocupados por servicio por cada egreso, $\mathrm{y}$

7. Indice de complejidad quirúrgica, definido por la frecuencia de los códigos de derechos de pabellón de los servicios respectivos, de acuerdo a los códigos del arancel del Fondo Nacional de Salud de Chile (FONASA).

Con los indicadores globales anteriormente mencionados, se construyó el índice de complejidad quirúrgica a partir de la frecuencia de códigos de derechos de pabellón, aceptando que este código es un indicador del nivel de complejidad del procedimiento quirúrgico efectuado, definiendo una escala creciente de complejidad, a través de un indicador ponderado, que en el caso del Hospital estudiado alcanzó un promedio de 3,62.

Se ha asumido que los códigos 1 y 2 representan un puntaje de complejidad 1; los códigos 3 y 4: 2; los códigos 5 y 6: 3; los códigos 7 y 8: 4; los códigos 9 y 10: 5; los códigos 11 y 12: 6; y los códigos 13 y 14: 7.

La matriz de datos obtenida (Tabla 1), ha sido tratada mediante la técnica multivariante descriptiva de análisis de componentes principales (ACP).

Estadística. El ACP es la técnica de análisis multivariante más antigua ${ }^{5,6}$. Sus aplicaciones prácticas se manifestaron cuando se desarrollaron los medios informáticos necesarios.
El ACP pretende simplificar un conjunto de datos, generalmente cuantitativos, procedentes de variables interrelacionadas. A partir de combinaciones lineales de las variables originalmente medidas, se obtiene un nuevo conjunto de igual número de variables, no correlacionadas, llamadas componentes principales (CP), en las cuales permanece la variabilidad presente en los datos originales. $\mathrm{Al}$ ordenarlas decrecientemente por su varianza, permiten explicar el fenómeno de estudio con las primeras $\mathrm{CP}^{7,8}$.

Con ello conseguimos: a. Sintetizar la información procedente de un volumen importante de datos; b. Crear nuevos indicadores o índices, representados por las CP, y c. Utilizar el ACP como paso previo a otras técnicas 9 .

En definitiva, tras aplicar el ACP, creamos nuevas variables, las CP. Pero, además, cada sujeto de la muestra (servicios médico-quirúrgicos hospitalarios) obtiene una puntuación en cada una de las $\mathrm{CP}$ seleccionadas, que permite resolver un problema frecuente en epidemiología, como el ordenamiento de sujetos cuando se tiene más de una medición de los mismos. Por otro lado, estas $\mathrm{CP}$ ayudan a desentrañar un modelo subyacente en el conjunto de datos iniciales.

En nuestro estudio hemos partido de la diagonalización de la matriz de correlaciones, ya que la interpretación de las componentes o factores, es más fácil cuando usamos variables estandarizadas. Los análisis se realizaron con el programa SAS. Tabla 1. D istribución de los valores de las variables por los servicios médico-quirúrgicos del
H ospital Clínico de la U niversidad de C hile, 2003

\begin{tabular}{|lrcrrcc|}
\hline Centro de Costo & Egresos & Letalidad & Reingresos & Consultas & Días camas ocupadas & $\begin{array}{c}\text { Complejidad } \\
\text { quirúrgica }\end{array}$ \\
\hline Cardiovascular & 1.178 & 0,2 & 8,48 & 5.017 & 6.159 & 5,71 \\
Cirugía & 2.663 & 0,1 & 6,0 & 11.145 & 12.008 & 3,93 \\
Neurocirugía & 1.209 & 0,4 & 8,27 & 4.026 & 7.845 & 5,25 \\
Oftalmología & 857 & 0,0 & 9,33 & 16.163 & 2.179 & 3,42 \\
Otorrinolaringología & 1.010 & 0,2 & 1,98 & 12.664 & 1.733 & 2,86 \\
Traumatología & 1.250 & 0,0 & 0,80 & 10.719 & 4.348 & 3,81 \\
Urología & 1.064 & 0,0 & 4,69 & 5.979 & 3.909 & 4,38 \\
Ginecología & 817 & 0,0 & 4,89 & 22.234 & 2.173 & 3,50 \\
Obstetricia & 1.929 & 0,0 & 0,51 & 13.275 & 6.686 & 3,49 \\
\hline
\end{tabular}




\section{RESUltados}

La matriz inicial de datos representada por las puntuaciones obtenidas por los diferentes servicios en las variables medidas, se puede observar en el Tabla 1. A continuación se obtuvo la matriz de correlaciones entre las variables, previa al proceso de diagonalización propio del ACP.

Se seleccionaron las dos primeras componentes, que en conjunto explican $75,92 \%$ de la variabilidad inicial. Para poder entender el significado de ambas componentes es necesario estudiar la correlación entre las variables originalmente medidas y las CP seleccionadas, que especifica la intensidad de la contribución de cada variable en la componente seleccionada y nos ayuda a nombrarla facilitando la interpretación del nuevo índice generado.

La matriz de correlaciones variables-componentes (Tabla 2), nos muestra que la correlación estadísticamente significativa de la primera componente se dio con la variable complejidad quirúrgica, seguida de la obtenida con la variable original días camas ocupadas, tasa de letalidad, reingresos y egresos. Se observó correlación negativa con la variable consultas externas.

En la segunda componente se obtuvo correlación significativa con la variable egresos, seguida por la obtenida con la variable días camas ocupados y consultas externas. Se oponen en ella la variable reingresos, la variable tasa de letalidad y la variable complejidad quirúrgica.
Procede a continuación calcular las puntuaciones que obtienen los servicios médico-quirúrgicos del hospital en las dos componentes seleccionadas, para poder dar un ordenamiento de los mismos. Los resultados obtenidos se pueden ver para todos los servicios médico-quirúrgicos en el Tabla 3. Observamos cómo en la primera componente los servicios médico-quirúrgicos con puntuación más alta y por orden fueron, el primero, neurocirugía; el segundo, cardiovascular y el

Tabla 2. C orrelación entre las variables originales y las dos componentes: primera componente, (complejidad asistencial), segunda componente, (demanda asistencial). H ospital Clínico de la Universidad de C hile, 2003

\begin{tabular}{|lcc|}
\hline & $11^{\underline{a}}$ & $2^{\underline{\underline{a}}}$ \\
& Componente & Componente \\
\hline Egresos & 0,34 & 0,92 \\
& 0,3642 & 0,0004 \\
Letalidad & 0,68 & $-0,31$ \\
Reingresos & 0,0425 & 0,4104 \\
& 0,52 & $-0,48$ \\
Consultas externas & 0,1441 & 0,1857 \\
& $-0,83$ & 0,09 \\
Días camas ocupados & 0,0048 & 0,8070 \\
& 0,71 & 0,67 \\
Complejidad quirúrgica & 0,0310 & 0,0469 \\
& 0,86 & $-0,28$ \\
& 0,0026 & 0,4544 \\
\hline
\end{tabular}

Tabla 3. 0 rden obtenido por los servicios para ambas componentes: primera componente, (complejidad asistencial). Segunda componente, (demanda asistencial). H ospital Clínico de la U niversidad de C hile, 2003

\begin{tabular}{|lclc|}
\hline Centro de Costo & 1a Componente & Centro de costo & 2a Componente \\
\hline Neurocirugía & 2,63 & Cirugía & 2,53 \\
Cardiovascular & 2,21 & Obstetricia & 1,75 \\
Cirugía & 1,34 & Traumatología & 0,45 \\
Urología & 0,05 & Urología & $-0,45$ \\
Obstetricia & $-0,81$ & Otorrinolaringología & $-0,53$ \\
Traumatología & $-0,91$ & Ginecología & $-0,61$ \\
Otorrinolaringología & $-1,18$ & Neurocirugía & $-0,91$ \\
Oftalmología & $-1,21$ & Cardiovascular & $-1,10$ \\
Ginecología & $-2,12$ & Oftalmología & $-1,11$ \\
\hline
\end{tabular}


tercero, cirugía. Al final se ubicaron otorrinolaringología, oftalmología y ginecología.

Son, por lo tanto, los servicios médico-quirúrgicos con una mayor complejidad asistencial los que ocuparon los primeros puestos, sin que esto lleve aparejada una mayor demanda por casos a atender.

Al estudiar la segunda componente, la clasificación aportada por la misma fue diferente, el servicio de cirugía fue el primero, en segundo lugar se encontró el servicio de obstetricia y el tercer lugar fue ocupado por traumatología. Los últimos puestos fueron ocupados, entre otros, por servicios médico-quirúrgicos que puntuaron mucho en la primera componente, como por ejemplo, neurocirugía y cardiovascular, sin embargo el último lugar lo ocupó oftalmología.

En esta segunda componente son los servicios médico-quirúrgicos con una mayor demanda asistencial los que suben en la clasificación, ocupando los últimos puestos servicios con patologías complejas dentro de su especialidad.

Observamos también cómo cirugía mantuvo el tercer puesto en la primera y el primer lugar en la segunda componente, lo que puede indicar una demanda importante, junto con casos relativamente complicados. La posición baja, puesto número ocho en la primera componente y el último lugar en la segunda componente del servicio de oftalmología, indica una demanda en número poco frecuente y también de baja complejidad, que habría que explicar por las características propias del hospital o por la existencia de otros servicios de oftalmología de elevada especialización que concentran un mayor número de casos, compitiendo con el servicio correspondiente del hospital.

Por último, para obtener una representación gráfica que clarifique lo expuesto realizamos, con las puntuaciones obtenidas de los servicios en las dos CP, un gráfico de dispersión (Figura 1) y un análisis por conglomerados ${ }^{10}$ utilizando el algoritmo jerárquico de Ward $^{11}$, que nos proporciona el dendograma de la Figura 2. En ella puede verse la clasificación de los servicios en grupos o conglomerados: a) Cardiovascular, neurocirugía y cirugía; b) Oftalmología y ginecología, y c) Otorrinolaringología, traumatología, obstetricia y urología.

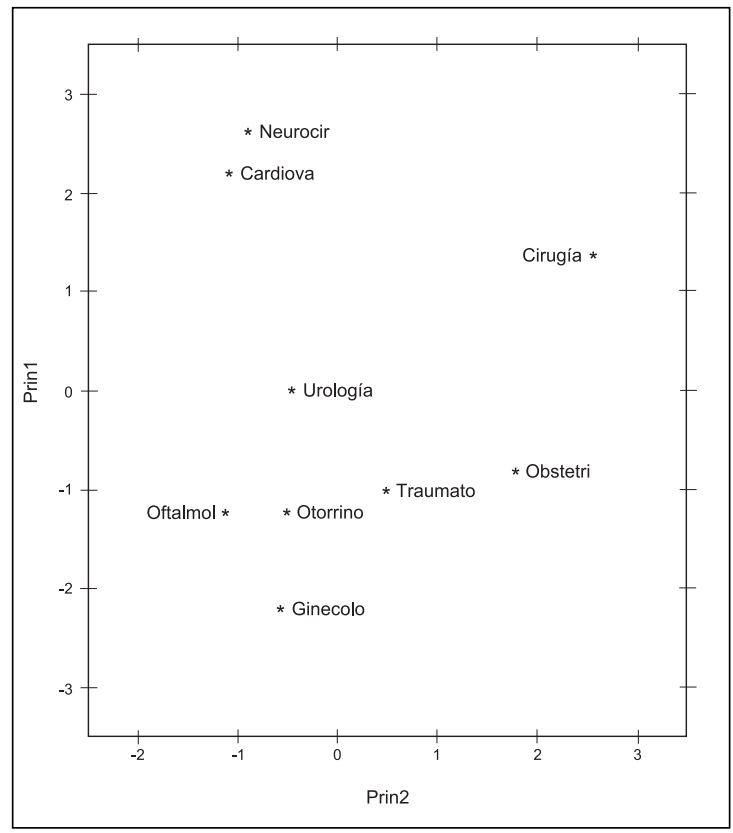

FiguRA 1. Gráfico de dispersión primera y segunda componentes principales. Hospital Clínico de la Universidad de Chile, 2003.

\section{DisCUSIÓN}

La actividad de un hospital se mide con indicadores como número de egresos, días camas ocupadas, promedio de días de estada, letalidad por servicios, tasa de reingresos y otros, a los cuales se unen indicadores que tienen en cuenta los casos atendidos, como son los derivados de los sistemas de clasificación de pacientes (SCP) como, por ejemplo, los grupos de diagnósticos relacionados (GDR).

Sin embargo, al no contar con GDR, nos hemos aventurado en esta etapa a definir nuestros propios indicadores clásicos y derivados de sistemas de clasificación de pacientes, entre los que se cuenta el índice de complejidad quirúrgica, definido a partir de los códigos de derechos de pabellón de los servicios respectivos de acuerdo al Arancel del Fondo Nacional de Salud de Chile (FONASA).

La definición de este último indicador de complejidad quirúrgica, que consideramos relevante para efectos de este análisis, nos hizo desechar en esta etapa la comparación con servi- 


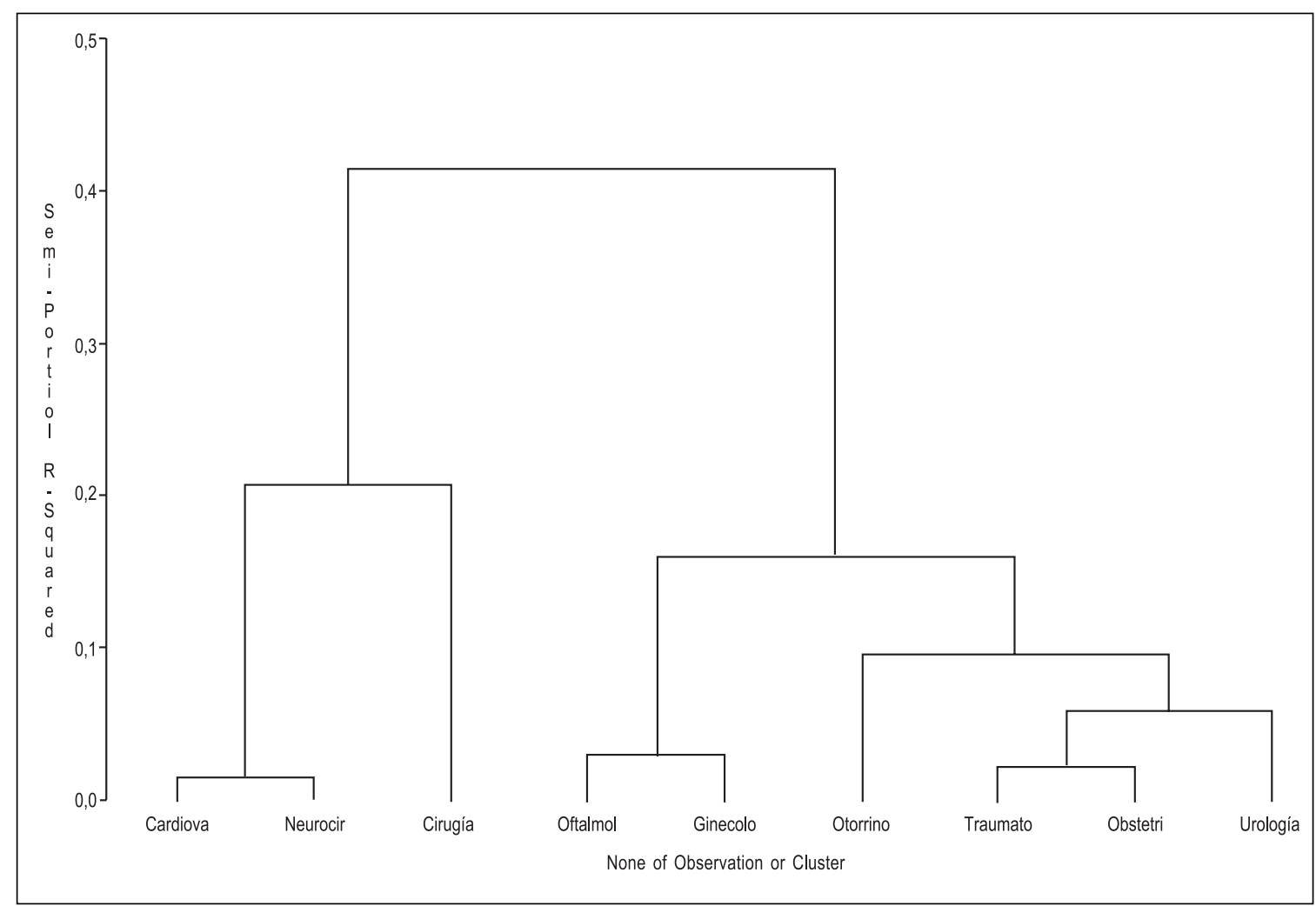

FiguRA 2. Puntuaciones de los servicios médico-quirúrgicos en las dos primeras componentes principales, año 2003.

cios médicos, sin actividad quirúrgica al interior del Hospital analizado.

Respecto de los resultados, hemos observado que la primera componente tiene una alta correlación con las variables complejidad quirúrgica, días camas ocupadas, tasa de letalidad, tasa de reingresos y número de egresos. Por el contrario, su correlación es negativa con la variable número de consultas externas.

La segunda componente principal obtiene su mayor correlación con las variables número de egresos, días camas ocupadas y consultas externas. Se oponen en ella las variables tasa de reingresos, tasa de letalidad y el índice de complejidad quirúrgica.

Debido a los pesos de las distintas variables en las dos componentes, y las puntuaciones de los diferentes servicios médico-quirúrgicos en las mismas, creemos que el ACP ha generado dos índices eficientes con el propósito de clasificar los servi- cios médico-quirúrgicos y plausibles para el modelo de hospital que monitorizamos, de tipo universitario.

Es interesante hacer ver cómo los servicios de neurocirugía y cardiovascular ocupan en la primera componente los primeros puestos y respecto a la segunda componente, el penúltimo y antepenúltimo puestos, lo que estaría señalando servicios en términos cualitativos, con una mayor complejidad de los casos tratados; pero con una baja presión asistencial en términos cuantitativos (poco número de pacientes). El servicio de cirugía respecto de la primera componente ocupa el tercer lugar en complejidad, pero el primer lugar en la segunda componente, demanda asistencial.

El servicio de oftalmología ocupa el penúltimo lugar en la primera componente asociada a complejidad asistencial y el último lugar respecto a la segunda componente asociada a la demanda 
asistencial, representando, por ende, un servicio de escasa demanda y complejidad.

Otro de los propósitos del ACP es utilizarlo como análisis previo a otras técnicas multivariantes. Para ello utilizamos las puntuaciones de los servicios en las dos componentes y hemos efectuado un gráfico de dispersión y un análisis de conglomerados que clarificara gráficamente los resultados.

En definitiva, el estudio conjunto multivariante que hacemos de los indicadores clásicos y derivados de sistemas de clasificación de pacientes mediante el ACP, complementa estudios que abordan el comportamiento de un solo indicador, ya sea la tasa de letalidad, la tasa de reingresos, los DRG, el porcentaje de casos extremos, etc., o de un conjunto de ellos pero a la manera univariante clásica ${ }^{12}$.

Consideramos que nuestro mayor aporte a la gestión de sistemas de salud con índices multivariantes, es desentrañar una dimensión subyacente e interpretable con los índices generados (complejidad y demanda) de fenómenos que se han medido de múltiples formas (indicadores univariantes clásicos: tasa de letalidad, tasa de reingre-

\section{REFERENCIAS}

1. Thomas JW, Ashcraft MLF. An evaluation of alternative severity of illness measures for use by university hospitals. Ann Arbor: Department of Health Services Management and Policy, School of Public Health, The University of Michigan, 1986.

2. THOMAS JW, AsHCRAFT MLF. Measuring severity of illness: A comparison of inter-rater reliability among severity methodologies. Inquiry 1989; 26 : 483-92.

3. Fetter RB, Shin Y, Freeman JL, AveriL RF, THOMpson JD. Case mix definition by Diagnosis Related Groups. Medical Care 1980; 18 (2 Suppl): 1-53.

4. Anuario Estadístico 2003. Hospital Clínico de la Universidad de Chile.

5. Pearson K. On lines and planes of closest fit to systems of points in space. Phil Mag 1901; 2: 559-72.

6. Hotewng H. Analysis of a complex of statistical variables into principal components. J Educ in Psychol 1933; 24: 417-41.

7. JowFFE IT. Principal Component Analysis. Nueva York (NY): Springer-Verlag, 1986. sos y otros), incapaces de incorporar toda la estructura del fenómeno y las interrelaciones entre indicadores, lo que creemos conseguir con los dos índices propuestos que, además, permiten ordenar por puntuaciones a los servicios médicoquirúrgicos teniendo en cuenta al unísono todos los aspectos útiles en los procesos de gestión.

Hasta el momento han sido pocos los trabajos que abordan la utilización del ACP en esta línea y sus aplicaciones más importantes son en campos tan dispares como la psicología, la educación, el control de calidad, la agricultura, la economía o la anatomía. Dentro de la epidemiología clínica y de la gestión hospitalaria pueden jugar un papel importante en la creación de índices como los presentados ${ }^{13,14}$.

Estos índices deberán ser validados definitivamente mediante su utilización en otros hospitales y en otros escenarios, lo que permitirá continuar con este tipo de investigaciones, especialmente en lo referente a la construcción de indicadores que sean propios a la realidad de cada establecimiento de salud y al grado de desarrollo sanitario en el cual se encuentran inmersos.

8. Almenara J, González JL, García C, Peña P. ¿Qué es el análisis de componentes principales? Jano 1998; 1268: 58-60.

9. GonzÁlez B. Análisis Multivariante. Aplicación al ámbito sanitario. Barcelona: SG Editores, 1991.

10. EveritT BS. Cluster Analysis. 3a ed. Londres: Edward Arnold, 1993.

11. WARD JH. Hierarchical grouping to optimize an objetive function. J American Statist Assoc 1963; 58: 236-44.

12. García C, Almenara J, García JJ. Proporción de casos extremos (outlier) por servicio como indicador de gestión. Rev Adm Sanit 1998; 8: 123-34.

13. Almenara C, García C, González JL, Abelián MJ. Creación de índices de gestión hospitalaria mediante análisis de componentes principales. Salud Pública Mex 2002, 44: 533-40.

14. Salinas H, Erazo M, Reyes A, Carmona S, Veloz P, MarTínez L Creación de índices de gestión de maternidades mediante el análisis de componentes principales. Rev Chil Obstet Ginecol 2004; 69: 24-34. 